CONGENITAL HEART DISEASE

\title{
Clinical course of fetal congenital atrioventricular block in the Japanese population: a multicentre experience
}

\author{
Y Maeno, W Himeno, A Saito, S Hiraishi, O Hirose, M Ikuma, N Inamura, M Kawataki, \\ A Mizukami, M Ota, H Shiraishi, G Satomi, H Kato
}

Heart 2005;91:1075-1079. doi: 10.1136/hrt.2003.033407

See end of article for authors' affiliations

.....................

Correspondence to: Dr Yasuki Maeno, Perinatal Care Centre, Department of Paediatrics, Kurume University School of Medicine, 67, Asahimachi, Kurume, 8300011, Japan; yasukim@ med.kurume-u.ac.jp

Accepted 21 October 2004

\begin{abstract}
Objectives: To elucidate the prenatal and postnatal course of fetal congenital atrioventricular block (CAVB) during the past decade in the Japanese population.

Design: Retrospective multicentre study. All fetuses with CAVB in 10 Japanese institutions in the period from January 1990 to August 2001 were included.

Patients: Of the 48 fetuses with CAVB, 17 had a congenital heart defect (CHD) (14 with left atrial isomerism) and 31 had a structurally normal heart ( 22 with positive maternal autoantibodies). Gestational age at diagnosis was 15 to 38 (median 26) weeks.

Results: Of the 17 fetuses with a CHD, three were aborted, one died before birth, and eight died after birth (three in the neonatal period and five after the neonatal period). Of the 31 fetuses without a CHD, two died before birth and two died after birth. CHD $(p=0.005)$ and the presence of fetal hydrops $(p=0.05)$ were significant risk factors for death. However, fetal ventricular and atrial heart rates, gestational age at delivery, and birth weight were not related to death. Transplacental medication of sympathomimetics increased the fetal heart rate in five of eight fetuses treated. Dexamethasone did not improve the degree of heart block in any of the six fetuses treated. Postnatally, pacemakers were implanted in 30 of 40 babies. Four fetuses with maternal autoantibodies had decreased cardiac function.

Conclusions: CHD and fetal hydrops are risk factors for prenatal and postnatal death. The fetal ventricular rate of 55 beats/min did not appear to be a threshold value by which to predict fetal hydrops. Patients with CAVB should be subjected to close long term follow up to check for the need for pacemaker implantation or for late onset cardiac dysfunction.
\end{abstract}

$\mathrm{F}$ etal echocardiography has become established as a method for detecting fetal arrhythmias. ${ }^{12}$ Some of the fetuses with either tachyarrhythmias or bradycardia develop hydrops and even die in utero. Successful intrauterine treatment leading to relatively benign prenatal and postnatal prognosis has been well documented in cases of fetal tachyarrhythmia, whereas intrauterine management and prognosis are still uncertain in cases of fetal bradycardia. ${ }^{1-7}$

Congenital atrioventricular block (CAVB) is the most common type of fetal bradycardia. ${ }^{1-7}$ Maternal autoantibodies, such as SSA and SSB antibodies, are the well known cause of CAVB. ${ }^{8}$ The incidence of this type of bradycardia has been reported in a range of one in 15000 to one in 20000 liveborn infants. ${ }^{6}$ Another well known cause of CAVB is a congenital heart anomaly. Left atrial isomerism, atrioventricular septal defect, and discordant atrioventricular connection are sometimes associated with CAVB..$^{1-3} 910$

Several studies of fetal CAVB to elucidate the prenatal and postnatal course and to search for better management have been published..$^{3-7}$ However, some of the risk factors for poor outcome vary between the reports. Current management strategies may change the impact of some of these risk factors. Recently, some postnatal complications, such as dilated cardiomyopathy and endocardial fibroelastosis, have been recognised in fetuses with isolated CAVB with autoantibodies. ${ }^{11-13}$ However, the incidence of these complications is unknown. Furthermore, the characteristics of fetal CAVB may vary with ethnicity. Hence, the objective of this study was to elucidate the prenatal and postnatal course and the outcome of fetal CAVB during the past 10 years in the
Japanese population and to find better management for the affected fetuses.

\section{METHODS}

This was a retrospective multicentre study. Questionnaires were sent to 10 Japanese institutions: Kurume University School of Medicine, Kurume; Osaka Medical Centre and Research Institute for Maternal and Child Health, Osaka; Kanagawa Children's Medical Centre, Yokohama; Shizuoka Children's Hospital, Shizuoka; Tokyo Women's Medical University, Tokyo; Matsuyama Red Cross Hospital, Matsuyama; Kitasato University, Sagamihara; Jichi Medical School, Utsunomiya; Hamamatsu University School of Medicine, Hamamatsu; and Seirei Hamamatsu General Hospital, Hamamatsu. All fetuses with bradycardia caused by CAVB and a fetal ventricular heart rate less than 100 beats/min during the period January 1990 to August 2001 were included in this study. The following prenatal data were collected for each patient: gestational age at time of diagnosis and delivery; presence or absence of congenital heart defect (CHD); presence or absence of maternal autoantibodies, such as SSA and SSB antibodies; presence or absence of fetal hydrops; fetal ventricular and atrial heart rate at presentation; minimum ventricular heart rate in utero; prenatal treatment; method of delivery; and outcome. Mild valvar pulmonary stenosis, patent ductus arteriosus, and atrial septal defect were categorised as absence of CHD. The postnatal course, cardiac function, follow up interval, and pacemaker implantation were also evaluated. The risk of fetal or neonatal death was analysed by multiple regression with a logistic model, and $\mathrm{p}<0.05$ was considered to be significant. 
Table 1 Characteristics of 48 fetuses with congenital atrioventricular block

\begin{tabular}{lllc}
\hline & Without CHD & With CHD & Total \\
\hline Number of patients & 31 & 17 & 48 \\
Gestational age at referral (weeks) & $25.3(13-36)$ & $26.5(17-38)$ & 26 \\
Fetal hydrops & 2 & 5 & 7 \\
Positive maternal autoantibodies & 22 & 0 & 22 \\
Ventricular HR at referral (beats/min) & $60.3(43-100)$ & $66.7(51-100)$ & 62.6 \\
Atrial HR at referral (beats/min) & $139.8(119-161)$ & $121.8(60-161)$ & 133.4 \\
\hline Data are median (range) or number of patients. & & \\
CHD, congenital heart defect; HR, heart rate. & & \\
\hline
\end{tabular}

\section{RESULTS}

Forty eight fetuses from 10 institutions fulfilled the criteria and their data were analysed (table 1). Gestational age at diagnosis was 15-38 weeks (median 26). Seventeen had CHD and 14 had left atrial isomerism (fig 1). There were no cases of discordant atrioventricular connection in this series. Thirty one fetuses had structurally normal hearts and 22 were positive for maternal autoantibodies (fig 2). All of the fetuses with positive antibodies were examined after 20 weeks. One of the fetuses without CHD had CAVB due to a cardiac tumour at the atrioventricular junction.

Of the 48 fetuses, 29 survived; three pregnancies were terminated (all three fetuses had left atrial isomerism but none had developed hydrops), three fetuses died in utero, three died in the neonatal period, seven died after the neonatal period, and three were lost to follow up. Fifteen babies were delivered vaginally and 25 were delivered by caesarean section (table 2). CHD $(p=0.005)$ and the presence of fetal hydrops $(\mathrm{p}=0.05)$ were significant risk factors for death (table 3). However, fetal ventricular and atrial heart rates, gestational age at delivery, and birth weight were not related to death.

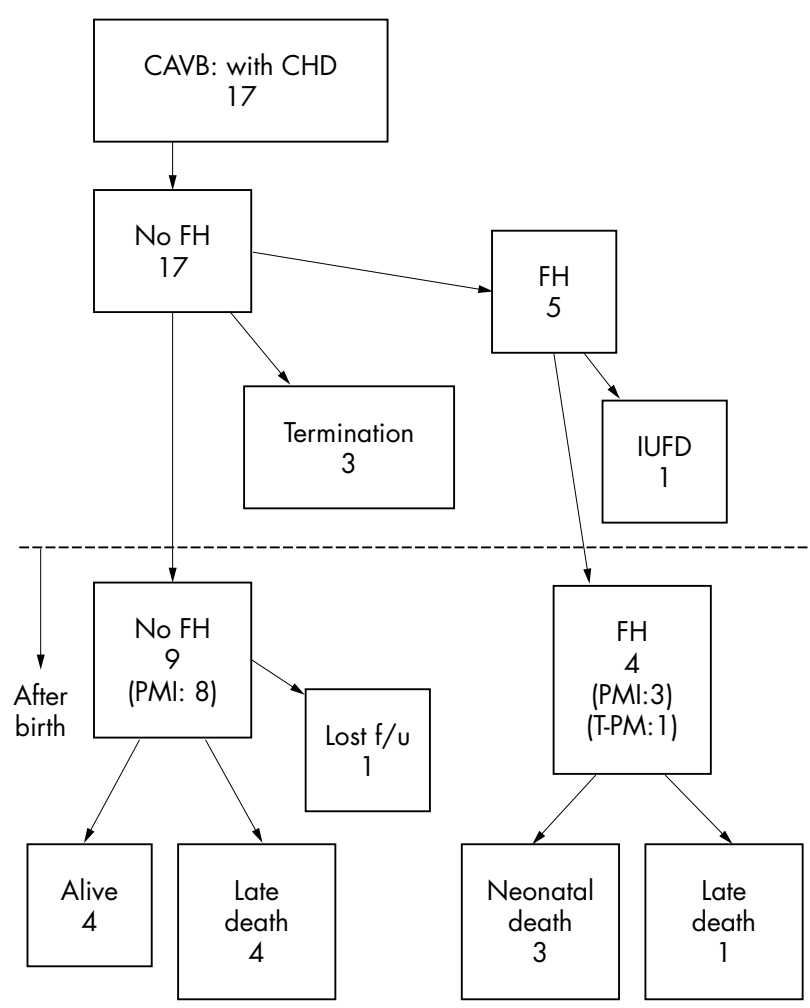

Figure 1 Prenatal and postnatal course of fetuses with congenital atrioventricular block (CAVB) with congenital heart defect (CHD). $\mathrm{FH}$, fetal hydrops; f/u, follow up; IUFD, intrauterine fetal death; PMI, pacemaker implantation; T-PM, temporary pacemaker.
Fetal hydrops was found in seven (five had CHD and two had normal cardiac morphology). Of the 17 fetuses with CHD, five developed fetal hydrops and all had left atrial isomerism. Their ventricular heart rates ranged from 55 to 67 beats/min (median 60). Of the 31 fetuses with normal cardiac morphology, two developed hydrops and both had maternal autoantibodies. The ventricular heart rates in the fetuses were 40 and 44 beats/min at the onset of fetal hydrops. Although 13 other fetuses with normal cardiac morphology (eight with positive maternal autoantibodies) had relatively low ventricular heart rates, at less than 55 beats/min (range 43 to 55 beats/min, median 50), they did not develop fetal hydrops.

Of the 22 fetuses positive for maternal autoantibodies, 20 were positive for SSA antibody. Of these 20, eight were also positive for SSB antibody. However, no fetus was positive for SSB antibody alone. Both of the fetuses negative for SSA antibody were positive for DNA antibody. Eight others were positive for DNA antibody as well as SSA antibody. Twelve mothers of the 22 fetuses had some symptoms of collagen disease (one mother had two fetal cases). However, only six mothers had a previous diagnosis of collagen disease. Sjogren's disease was diagnosed in six mothers, systemic lupus erythematosus in three, both systemic lupus erythematosus and Sjogren's disease in one, and Hashimoto's disease in two.

Transplacental medication was administered to 16 fetuses (table 2). Sympathomimetics were administered to the mother in eight cases in an attempt to increase fetal heart rate, with the result that the fetal ventricular heart rate increased by more than $10 \%$ in five of the nine fetuses. One fetus with left atrial isomerism and fetal hydrops was treated by continuous maternal infusion of sympathomimetics (ritodrine) beginning at 31 weeks of gestation. The fetal ventricular heart rate increased from 61 to 72 beats/min and fetal ascites decreased. Dexamethasone was administered to six mothers and was instituted from 20-28 weeks of gestation. However, no fetus had an improved degree of heart block. No fetus was treated with an invasive intrauterine procedure such as pacemaker implantation.

Postnatal follow up data were obtained for 39 fetuses (three were lost follow up). The follow up period ranged from 0 days to 10 years (mean 2.4 years). Thirty babies had a pacemaker implanted. Of these 30, 19 received a pacemaker within the first postnatal week and five others underwent the procedure within the first month of life. However, the number of patients with pacemaker implantation continued to increase during long term follow up. Three had a pacemaker implanted within one year of life, one received a pacemaker at 18 months, one at 19 months, and one at five years after birth. All these babies had had more than one of the following indications for pacemaker implantation as recommended by the Japanese guidelines: syncope, ventricular heart rate of less than 50 beats/min, decreased cardiac function, and sudden pause longer than 2-3 times the regular ventricular heart rate. All 12 fetuses with CHD who survived 


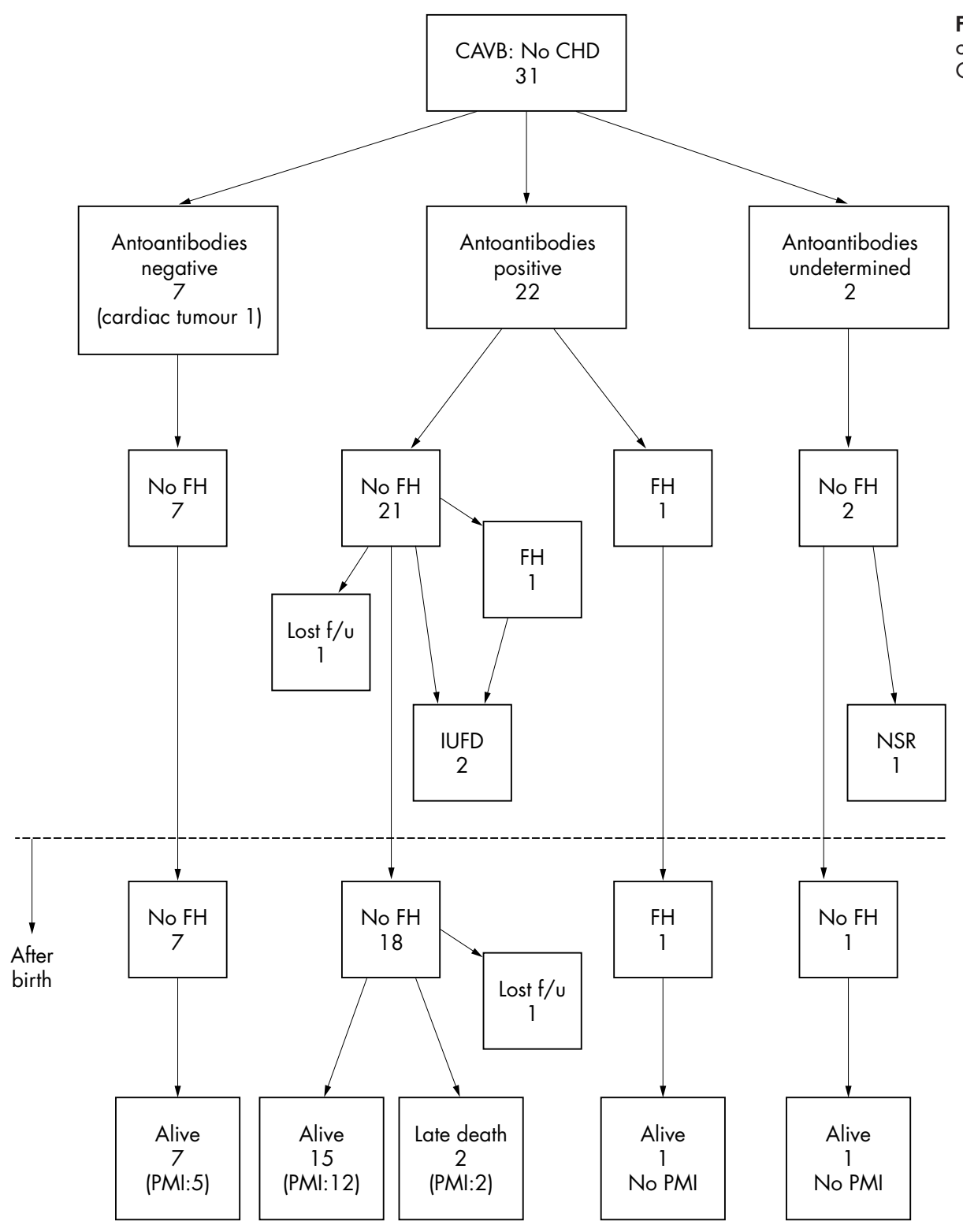

Figure 2 Prenatal and postnatal course of fetuses with CAVB without CHD. NSR, normal sinus rhythm.

the prenatal period received a pacemaker and all 16 fetuses with a ventricular heart rate of less than 60 beats/min during the prenatal period had a pacemaker implanted. Four fetuses with normal cardiac morphology had decreased cardiac function and all had maternal autoantibodies. All had a pacemaker implanted but two died postnatally. Three of the four were noted to have decreased cardiac function prenatally and one died. Two underwent myocardial biopsy and histological analysis of both showed myocardial hypertrophy,

\begin{tabular}{|c|c|c|}
\hline & Without CHD & With CHD \\
\hline \multicolumn{3}{|l|}{ Intrauterine treatment } \\
\hline Sympathomimetics & 5 & 3 \\
\hline Steroids & 6 & 0 \\
\hline \multicolumn{3}{|l|}{ Method of delivery } \\
\hline Caesarean section & 18 & 7 \\
\hline Vaginal & 9 & 6 \\
\hline
\end{tabular}

compatible with dilated cardiomyopathy. The other fetus developed cardiac dysfunction at 18 months postpartum and died at 28 months. Postnatal pathological analysis showed endocardial fibroelastosis.

In one fetus, two to one heart block at 19 weeks of gestation reverted to sinus rhythm before birth. Review of the videotape of the fetal echocardiogram at 19 weeks of gestation showed that fetal atrial contraction was regular with a rate of 140 beats/min and fetal ventricular heart rate

Table 3 Risk for death: multiple regression by logistic model

\begin{tabular}{llll}
\hline & $\mathbf{p ~ V a l u e}$ & $\mathbf{R}$ & Odds ratio \\
\hline CHD & 0.0046 & 0.33 & 24.75 \\
Fetal hydrops & 0.0451 & 0.17 & 5.65 \\
Gestational age & 0.4002 & 0 & 0.73 \\
Birth weight & 0.2050 & 0 & 0.99 \\
Delivery method & 0.9378 & 0 & 1.09 \\
\hline
\end{tabular}


was 70 beats/min. The fetus had normal cardiac morphology. Maternal autoantibodies were not assessed in this case. Although this fetus was not considered to require intrauterine treatment, the mother received ritodrine to control uterine contraction. The day after ritodrine was started, the fetus developed a normal sinus rhythm. Postnatal ECG showed a normal PR interval and normal QT interval.

\section{DISCUSSION}

Congenital heart block is the most common type of fetal bradycardia. ${ }^{2-7}$ Several studies of fetal CAVB to elucidate the prenatal and postnatal course and to search for better management have been published. Maternal autoantibodies, such as SSA and SSB antibodies, are the well known cause of a rare form of CAVB. Another well known cause of CAVB is $\mathrm{CHD}$, such as left atrial isomerism, atrioventricular septal defect, and discordant atrioventricular connection. ${ }^{23910}$ However, management of fetal bradycardia is an ongoing dilemma.

Several issues need to be addressed in fetal CAVB. Risk factors for poor outcome vary between reports and are still unclear. $^{2-7}$ Recently, some postnatal complications, such as a dilated cardiomyopathy and endocardial fibroelastosis, have been recognised in cases of CAVB with autoantibodies. ${ }^{11-13}$ However, detailed information such as the incidence and natural history of these complications is rarely available. Furthermore, recently developed fetal heart screening procedures and management strategies such as intrauterine treatment may alter the clinical course of fetal CHD. In addition, the characteristics of fetal CAVB in the Japanese population may differ from those of patients in previously published reports. Hence, we selected cases of fetal CAVB in the past 10 years from Japanese institutions and attempted to answer these questions.

Although the presence of CHD and fetal hydrops were significant risk factors for intrauterine death or neonatal death in fetal CHD, low fetal ventricular heart rate did not appear to be a significant risk factor in our study. Several earlier studies noted that a ventricular heart rate of less than 55 beats/min was a risk factor for death. ${ }^{3}{ }^{4}$ However, Jaeggi and others ${ }^{5}$ recently reported that fetuses with CAVB without CHD and with a ventricular heart rate of less than 55 beats/ min were not at risk. Other case reports also documented uncomplicated prenatal courses in fetuses with extremely low heart rates with CAVB without CHD. ${ }^{14}{ }^{15}$ Jaeggi and colleagues $^{5}$ speculated that recent perinatal management, in particular intrauterine treatment, altered the former risk factors. We also believe that analysis of risk factors in a heterogeneous population, as performed in earlier studies, poses problems. The threshold of 55 beats/min as a risk for death appears too low for fetuses with CHD. In the present study, all of the fetuses with CAVB with CHD who developed fetal hydrops had a fetal heart rate greater than 55 beats/min. On the other hand, the threshold of 55 beats/min appears too high for fetuses without CHD. According to our study, fetuses with normal cardiac morphology tolerated well a fetal heart rate of less than 55 beats/min. Only two fetuses with extremely low heart rates of less than 44 beats/min developed hydrops. Although our results appear to indicate that low ventricular rate is not a significant risk factor for death, the number of patients was insufficient to support this conclusion and studies of larger numbers of fetuses are required.

Our study failed to identify any risk factors for fetuses with normal cardiac morphology; they neither developed hydrops nor had a poor outcome. After reviewing individual clinical histories, we hypothesise that poor cardiac function or the presence of atrioventricular valve insufficiency may be important in the development of fetal hydrops and in prenatal and postnatal death. However, these factors were not always identified in each study; therefore, they could not be included in our analysis. Prospective studies with serial measurements of cardiac function are indicated to clarify these risk factors.

Several studies have shown both the efficacy and difficulty of intrauterine treatment of fetuses with CAVB. ${ }^{3516-19}$ Our results suggest that maternal administration of sympathomimetics such as ritodrine or salbutamol is effective treatment to increase the fetal ventricular heart rate. The fetal ventricular heart rate increased by more than $10 \%$ in five of eight fetuses and fetal hydrops resolved in one. Sympathomimetics may be a reasonable option for use in fetuses with a lower ventricular heart rate and with early signs of hydrops; these drugs may prevent the development of severe hydrops and decrease the need for preterm delivery. ${ }^{16}{ }^{17}$ Conversely, our retrospective study did not find any benefit in using steroids to treat CAVB secondary to maternal autoantibodies. A previous study showed that maternal administration of dexamethasone improved the degree of heart block in some fetuses with CAVB. ${ }^{17}$ In that study, the authors hypothesised that early administration after the development of heart block is important for successful treatment. In our study steroids were administered later, which may be one of the reasons that the treatment was not effective. Nevertheless, intrauterine administration of dexamethasone is thought to have another benefit for fetuses with maternal autoantibodies. The maternal autoantibodies are known to cause fetal myocarditis and endocardial fibroelastosis, which lead to severe cardiac dysfunction in both the prenatal and postnatal periods. ${ }^{18} 19$ Dexamethasone may resolve or prevent cardiac dysfunction, but it was difficult to prove this effect in our retrospective study.

One fetus with two to one atrioventricular block reverted to sinus rhythm before birth without any intrauterine treatment. At the time of data collection for this multicentre study, two fetuses had been reported to have had spontaneous recovery of sinus rhythm before birth. Two authors reviewed the videotapes of fetal echocardiography in these cases. One case was diagnosed as blocked atrial bigeminy, hence excluded from this study. $M$ mode echocardiography in the other case showed regular atrial contraction; hence, the diagnosis of CAVB was confirmed. Unfortunately, the cause of CAVB in this fetus was not determined. Nevertheless, the possibility of spontaneous recovery should be considered during the management of the fetuses with CAVB without CHD.

Our study showed a high incidence of pacemaker implantation in babies with prenatal onset of CAVB. This finding correlates with several previous studies. ${ }^{3-5}$ All babies with CHD required a pacemaker to increase cardiac output. Structurally abnormal hearts may have a lower threshold for bradycardia than structurally normal hearts. Since the majority of the fetuses had left atrial isomerism and needed a single ventricular-type repair, their CAVB is one of the significant long term risk factors. Conversely, structurally normal hearts did not always require a pacemaker. Some fetuses have relatively high heart rates before and after birth. However, our study showed that the incidence of pacemaker implantation continuously increased during follow up. Hence, close long term follow up is warranted.

The late onset of cardiac dysfunction and endocardial fibroelastosis has been recognised as one of the important complications of fetal CAVB. ${ }^{11-13}$ In our study three fetuses had cardiac dysfunction and one had endocardial fibroelastosis. All fetuses had normal cardiac morphology and positive maternal autoantibodies. The incidence is relatively high: three of 22 and one of 22 , respectively. 


\section{Conclusion}

CHD and fetal hydrops are the risk factors for prenatal and postnatal death. The likelihood of requiring a pacemaker was very high in both babies with CHD and those with a ventricular rate less than 60 beats/min at the prenatal evaluation. Patients with CAVB should receive close long term follow up to check for the need for a pacemaker and to detect late onset cardiac dysfunction. Fetuses with CAVB and normal cardiac morphology rarely develop hydrops even when the ventricular rate is less than 50 beats/min. Fetuses with both CAVB and CHD develop hydrops even when the ventricular rate is more than 55 beats $/ \mathrm{min}$. The anatomical problems such as valve regurgitation seem to be a more important risk factor for these fetuses.

\section{ACKNOWLEDGEMENTS}

This study was conducted by the Multi-Institutional Study Committee of the Japanese Society of Fetal Cardiology.

\section{Authors' affiliations}

Y Maeno, W Himeno, H Kato, Kurume University School of Medicine, Kurume, Japan

A Saito, Shizuoka Children's Hospital, Shizuoka, Japan

S Hiraishi, Kitasato University, Sagamihara, Japan

O Hirose, Matsuyama Red Cross Hospital, Matsuyama, Japan

M Ikuma, Hamamatsu University School of Medicine, Hamamatsu, Japan

N Inamura, Osaka Medical Centre and Research Institute for Maternal and Child Health, Osaka, Japan

M Kawataki, Kanagawa Children's Medical Centre, Yokohama, Japan A Mizukami, Seirei Hamamatsu General Hospital, Hamamatsu, Japan M Ota, Tokyo Women's Medical University, Tokyo, Japan

H Shiraishi, Jichi Medical School, Utsunomiya, Japan

G Satomi, Nagano Children's Hospital, Nagano, Japan

\section{REFERENCES}

1 Simpson J. Fetal arrhythmias. In: Allan L, Hornberger LK, Sharland G, eds. Textbook of fetal cardiology. London: Greenwich Medical Media 2000:423-37.
2 Rosenthal E. Fetal heart block. In: Allan L, Hornberger LK, Sharland G, eds. Textbook of fetal cardiology. London: Greenwich Medical Media, 2000:438-52.

3 Schmidt KG, Ulmer HE, Silverman $\mathrm{NH}$, et al. Perinatal outcome of fetal complete atrioventricular block: a multicenter experience. J Am Coll Cardiol 1991;91:1360-6.

4 Groves AMM, Allan LD, Rosenthal E. Outcome of isolated congenital complete heart block diagnosed in utero. Heart 1996;75:190-4.

5 Jaeggi ET, Hamilton RM, Silverman ED, et al. Outcome of children with fetal, neonatal or childhood diagnosis of isolated congenital atrioventricular block. J Am Coll Cardiol 2002;39:130-7.

6 Buyon JP, Hiebert R, Copel J, et al. Autoimmune-associated congenital heart block: demographics, mortality and recurrence rates obtained from national neonatal lupus registry. J Am Coll Cardiol 1998;31:1658-66.

7 Frohn-Mulder IM, Meilof JF, Szatmari A, et al. Clinical significance of maternal anti-Ro/SS-A antibodies in children with isolated heart block. J Am Coll Cardiol 1994;23:1677-81.

8 Ho SY, Esscher E, Anderson RH, et al. Anatomy of congenital complete heart block and relation to maternal anti-Ro antibody. Am J Cardiol 1986;58:291-4

9 Gilljam T, McCrindle BW, Smallhorn JF, et al. Outcome of left atrial isomerism over a 28 -year period at a single institution. J Am Coll Cardiol 2000;36:908-16

10 Ho SY, Fagg N, Anderson RH, et al. Disposition of the atrioventricular conduction tissues in the heart with isomerism of the atrial appendages: its relation to congenital complete heart block. J Am Coll Cardiol 1992;20:904-10.

11 Moak JP, Barron KS, Hougen TH, et al. Congenital heart block: development of late-onset cardiomyopathy, a previous underappreciated sequela. J Am Coll Cardiol 2001;37:238-42.

12 Udink ten Cate FEA, Breur JMPJ, Cohen MI, et al. Dilated cardiomyopathy in isolated congenital complete atrioventricular block: early and long-term risk in children. J Am Coll Cardiol 2001;37:1129-34.

13 Nield LE, Silverman ED, Taylor GP, et al. Maternal anti-Ro and anti-La antibody-associated endocardial fibroelastosis. Circulation 2002;105:843-8.

14 Singh GK, Shumway JB, Amon E, et al. Role of fetal echocardiography in the management of isolated fetal heart block with ventricular rate $<55$ beats $/ \mathrm{min}$. Am J Perinatol 1998;15:661-8.

15 Minassian VA, Jazayeri A. Favorable outcome in a pregnancy with complete fetal heart block and severe bradycardia. Obstet Gynecol 2002;100:1087-9.

16 Groves AMM, Allan LD, Rosenthal E. Therapeutic trial of sympathomimetics in three cases of complete heart block in the fetus. Circulation 1995:92:3394-6.

17 Copel JA, Buyon JP, Kleinman CS. Successful in utero therapy of fetal heart block. Am J Obstet Gynecol 1995;173:1384-90

18 Rosenthal D, Druzin M, Chin C, et al. A new therapeutic approach to the fetus with congenital complete heart block: preemptive, targeted therapy with dexamethasone. Obstet Gynecol 1998:92:689-91.

19 Shinohara K, Miyagawa S, Fujita T, et al. Neonatal lupus erythematosus: results of maternal corticosteroid therapy. Obstet Gynecol 1999;93:952-7.

\section{IMAGES IN CARDIOLOGY}

\section{Intravascular ultrasound imaging of separate ostium of left anterior descending and circumflex} arteries

\begin{abstract}
A 62 year old woman with a history of hypertension and atypical chest pain was referred for coronary angiography following an abnormal exercise treadmill stress test. Coronary angiography showed separate ostium of the left anterior descending (LAD) and left circumflex arteries and apparent stenosis of the ostial portion of the LAD. Intravascular ultrasound (IVUS) imaging (Galaxy II, Boston Scientific Corp, Natick, Massachusetts, USA) showed an oval orifice of the LAD measuring $9 \mathrm{~mm}^{2}$ in cross sectional area with no atherosclerosis. IVUS assessment of the LAD in the setting of separate ostium can be useful to exclude significant atherosclerosis.
\end{abstract}

J Thomas

D M Shavelle dshavelle@hotmail.com

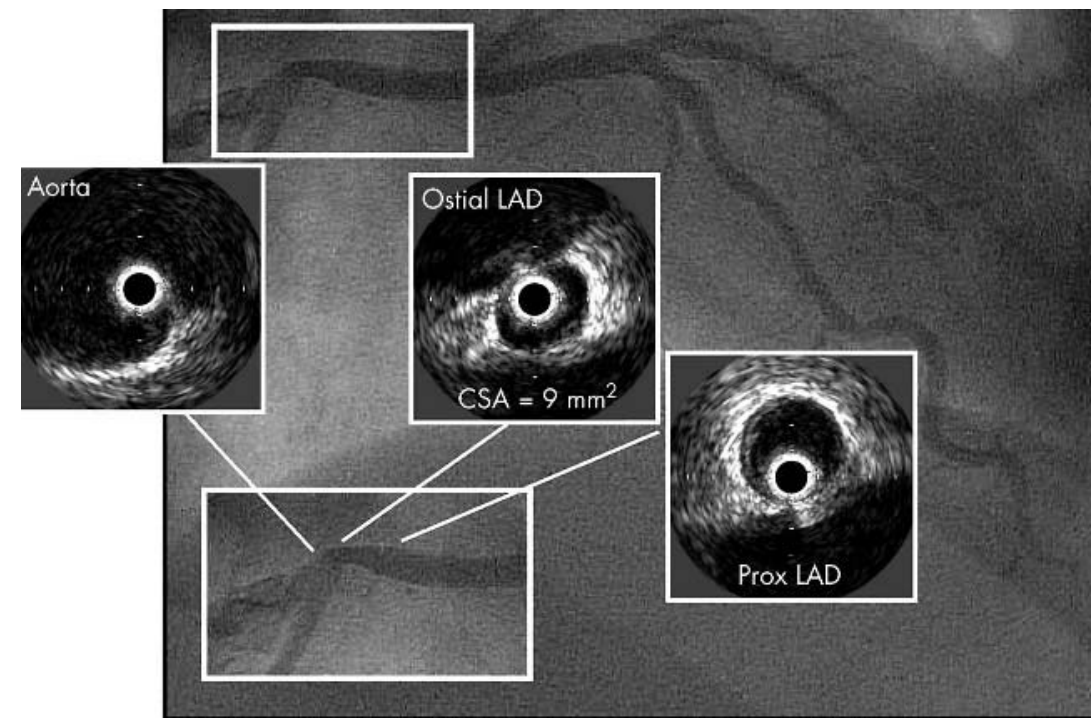

